\title{
Effectiveness of mobile applications to quit smoking: Systematic review and meta-analysis
}

\author{
Raquel Cobos-Campos ${ }^{1}$, Arantza Sáez de Lafuente ${ }^{1}$, Antxon Apiñaniz ${ }^{1,2}$, Naiara Parraza ${ }^{1}$, Iraida Pérez Llanos ${ }^{1,3}$, Gorka \\ Orive ${ }^{4,5,6,7}$
}

\begin{abstract}
INTRODUCTION Tobacco is the leading cause of preventable mortality. The use of mobile phones has grown exponentially, becoming a powerful tool to be used in health care.

METHODS In order to assess the effectiveness of mobile phones to quit smoking, we have carried out a systematic review and meta-analysis of randomized clinical trials evaluating interventions based on mobile applications for smartphones, that were not a smaller version of the same application, against other types of therapy. To address this, a bibliographic search was carried out in MEDLINE, EMBASE and COCHRANE LIBRARY. To obtain the combined effect, the relative risk and the $95 \%$ confidence interval were used. A heterogeneity and sensitivity analysis were also conducted.

RESULTS A total of nine studies were identified, but five were excluded. Qualitative review was performed with four selected studies, but quantitative analysis was carried out for only three, given the impossibility of calculating the RR in one of the studies. After combining the results, an RR of 0.901 (95\% CI: 0.57-1.423) was calculated comparing the effectiveness of mobile applications versus others type of interventions. This measure was robust, as shown by the sensitivity analysis.

CONCLUSIONS According to the results, it cannot be concluded that apps are effective for quitting tobacco. There are very few clinical trials published evaluating the effectiveness of mobile applications compared to other alternatives. Several clinical trials are still in progress, therefore their results have not been included in the present meta-analysis.
\end{abstract}

\author{
AFFILIATION \\ 1 Bioaraba Health Research Institute, Epidemiology \\ and Public Health research group, Vitoria-Gasteiz, \\ Spain \\ 2 Osakidetza Basque Health Service, Lakuabizkarra \\ Health Centre, Vitoria-Gasteiz, Spain \\ 3 Osakidetza Basque Health Service, Olaguibel Health \\ Centre, Vitoria-Gasteiz, Spain \\ 4 School of Pharmacy, Laboratory of Pharmaceutics, \\ University of the Basque Country UPV/EHU, Vitoria- \\ Gasteiz, Spain \\ 5 Bioaraba Health Research Institute, Nanobiocel \\ research group, Vitoria-Gasteiz, Spain \\ 6 University Institute for Regenerative Medicine \\ and Oral Implantology, Foundation Eduardo Anitua, \\ Vitoria-Gasteiz, Spain \\ 7 Singapone Eye Research Institute, Singapore, \\ Singapore
}

\section{CORRESPONDENCE TO}

Raquel Cobos-Campos. Bioaraba Health Research Institute, Isabel Orbe street, w/n, 01002, VitoriaGasteiz, Spain. E-mail: raquel.coboscampos@ osakidetza.eus

Gorka Orive. School of Pharmacy, Laboratory of Pharmaceutics, NanoBioCel Group, University of the Basque Country, Paseo de la Universidad 7, VitoriaGasteiz, 01006, Spain. E-mail: gorka.orive@ehu.eus

\section{KEYWORDS}

smoking cessation, mobile applications MeSH Unique ID: D063731, Telemedicine MeSH Unique ID: D017216

\section{Received: 24 April 2020}

Revised: 25 June 2020

Accepted: 23 September 2020

\section{INTRODUCTION}

Tobacco is the leading cause of preventable mortality ${ }^{1-3}$ and one of the biggest threats to public health. It kills at least 8 million people a year, of which more than 7 million are direct consumers and around 1.2 million are non-smokers exposed to the smoke of others. Unfortunately, reducing smoking consumption is not enough. In those smokers who reduce their consumption by more than $50 \%$, the risk of mortality from all causes is similar to those who continue smoking ${ }^{4}$. In addition, despite worldwide awareness campaigns about the risks of smoking, not 
many people understand the specific health risks of inhalation. For example, a study conducted in China in 2009 revealed that only $38 \%$ of smokers knew that tobacco causes coronary heart disease, and only $27 \%$ knew that it provokes strokes ${ }^{5}$.

Quitting smoking can be a particular challenge. According to a study, over 30 attempts to quit may be necessary to quit smoking ${ }^{6}$. Nicotine dependence is a complex disorder. In fact, it has been observed that the greater the nicotine dependence, the lower the probability of success ${ }^{7}$, motivation being one of the main factors to quit the habit ${ }^{8}$. As a consequence, development and optimization of behavioral strategies to quit smoking are gaining relevance.

Behavioral support strategies increase dropout rates $^{9}$. In the last few years, a wide range of platforms and strategies of behavioral therapy based on clinical-patient health advice have been developed including the telephone line, text messaging, webbased interventions and mobile applications that can be used independently or as an adjuvant tool ${ }^{10}$. Whittaker et al. ${ }^{11}$ published in 2016 a meta-analysis of interventions based on mobile telephones to quit smoking that included all the tools described above except mobile applications (apps). Results showed a beneficial effect compared to usual clinical practice $(\mathrm{RR}=1.67 ; 95 \% \mathrm{CI}: 1.46-1.90)^{11}$. Other research studies have found similar results ${ }^{12-14}$. On the other hand, applications to quit smoking have also been developed through mobile phones, though data on this matter are still limited ${ }^{15}$. In fact, data on the quality of content and the effectiveness of the approach are still scarce ${ }^{16-18}$. For instance, in a recent systematic review, although authors identified almost 50 mobile applications for smoking cessation, only $4 \%$ had suitable scientific support ${ }^{19}$.

Since the launch of mobile networks in the 1980 s, the use of mobile phones has grown exponentially. The International Communications Union estimated that by the end of 2015 there would be about 7 billion mobile phones worldwide, that is a penetration of $97 \%$, making these devices increasingly useful for healthcare ${ }^{20}$. Indeed, numerous applications are now available to provide help with many important tasks including information and time management, maintenance and access to sanitary records, communications and consulting, reference and information gathering, patient management and monitoring, clinical decision making and medical education and training $^{21-23}$.

The use of health applications among mobile phone users has also increased significantly. In a survey conducted in the United States, it was concluded that a little over half $(58.23 \%)$ of mobile phone users had downloaded a health-related mobile app. These results were dependent on several factors such as age and educational level, with the age of app users being lower ( $\mathrm{OR}=0.97$; 95\% CI: 0.96-0.98) than that of non-users, and the higher educational level related to higher app usage $(\mathrm{OR}=1.12 ; 95 \% \mathrm{CI}$ : 1.01-1.24 $)^{24}$. However, in another study evaluating the characteristics of users of mobile applications to quit smoking, it was found that demographic characteristics such as age and education level were not associated with the attitude towards the use of a tobacco cessation application, but rather depended on characteristics related to the content of the application instead of the general characteristics of the users ${ }^{25}$.

Assuming the wide penetration of mobile telephones and the use of health applications by the population (3.2 billion downloads in 2016) $)^{26}$, we decided to explore whether the use of smoking cessation applications is supported by scientific evidence. To address this issue, a systematic review and meta-analysis of randomized clinical trials evaluating interventions based on mobile applications to quit smoking was carried out.

\section{METHODS}

We conducted a systematic review and meta-analysis of clinical trials evaluating the effectiveness of applications for smartphones to quit smoking against other types of therapy that were not a smaller version of the same application, or other different applications (SMS, computer or tablet websites, clinical practice, health advice on smoking). For this purpose, we performed, in December 2019, first a bibliographic search, in MEDLINE, EMBASE and COCHRANE LIBRARY databases with the following search string: ('Mobile Applications' [Mesh]) AND ('Smoking Cessation' [Mesh]), filtering by type of study including Clinical Trial, Meta-Analysis, Randomized Controlled Trial, and Systematic Review. No language restriction was applied. Given the lack of studies, we 
also searched in google and other web browsers, and searched for unpublished studies in the platform clinicaltrials.gov.

The studies could be masked and not masked. The main variable evaluated was smoking abstinence. The follow-up period was between 3 and 6 months.

A qualitative and quantitative analysis was performed with selected studies. In the qualitative analysis, risk of bias assessment was carried out (random sequence generation of allocation, concealment of allocation, blinding of participants and staff, blinding of outcome assessors, incomplete outcome data, and selective reporting of results). In the quantitative analysis the risk of publication bias, heterogeneity test, estimation of pooled measure and a sensitivity analysis were performed.

\section{Quality assessment of clinical trials}

An evaluation of the risk of bias of the different studies included was carried out using the tool developed by the Cochrane group, which is a domain-based evaluation (random sequence generation of allocation, concealment of allocation, blinding of participants and staff, blinding of outcome assessors, incomplete outcome data, selective reporting of results, other biases $)^{27}$. Each domain is classified as high, medium or low risk of bias. All studies were included, regardless of the risk of each ${ }^{27}$.

\section{Heterogeneity analysis}

A heterogeneity analysis was performed to evaluate the variability among the studies included in the meta-analysis. To address this, the statistical test $\mathrm{Q}$ of DerSimonian and Laird ${ }^{28}$ was used. Due to low statistical power, the confidence level was set at $90 \%{ }^{29}$. The Q test was completed with the graphics of Galbraith $^{30}$ and L'Abbé et al. ${ }^{31}$. The $\mathrm{I}^{2}$ index was also calculated from the equation:

$\mathrm{I}^{2}=(\mathrm{Q}-\mathrm{df}) / \mathrm{Q} \times 100 \%$

where $Q$ is the Cochran homogeneity test statistic and $\mathrm{df}$ is the degrees of freedom (number of trials minus 1 ). The heterogeneity was considered important if $I^{2}$ $>50 \%{ }^{32}$.

\section{Publication bias}

Possible publication bias was assessed using the Egger and Begg statistic and two graphs (Funnel plot and Egger) were constructed. The confidence level for both tests was set at $95 \%$.

\section{Pooled measured estimation}

Tobacco cessation was measured by the variable 30 days (30PP) or 7 days point prevalence (7PP) at 6 months from the beginning. To obtain the combined effect for all the studies included in the meta-analysis, the relative risk (RR) and the 95\% confidence interval were used.

\section{Sensitivity analysis}

The influence of each of the studies on the overall estimation of the effect and, therefore, the robustness of the final measurement, was studied. To address this, the meta-analysis was repeated as many times as the number of the selected studies, skipping one of the studies each time while combining the remaining ones.

\section{RESULTS}

A total of nine studies were identified for this meta-analysis. After a more thorough review of the identified studies by the research team, five were discarded because they did not meet the eligibility criteria. In fact, four of them compared different versions of the same application (one more extensive version and another much smaller), and 1 was not a clinical trial but a cross-sectional study evaluating the characteristics of users of web-based programs or websites for mobiles (Figure 1). The characteristics of the four studies ${ }^{33-36}$ selected are summarized in Table 1. Qualitative analysis was performed with four studies, but the quantitative analysis was only carried out with the studies of Buller et al. ${ }^{35}$, Danaher al. ${ }^{34}$, and Baskerville et al. ${ }^{36}$, given the impossibility of calculating the RR for the study by Peiris et al. ${ }^{33}$.

\section{Risk assessment of bias}

The risk assessment of bias of each study is analyzed in Figure 2. The main characteristics of each study are now described.

In the study of Peiris et al. $.^{33}, 49$ current Aboriginal smokers (aged $>16$ years), who were willing to make a quit attempt in the next month, and with access to an iPhone or Android smartphone participated in the clinical trial. The intervention consisted of a personalized profile and quit plan, text and inapp motivational message. The comparator was usual cessation support services. Randomization 
Figure 1. PRISMA flow diagram
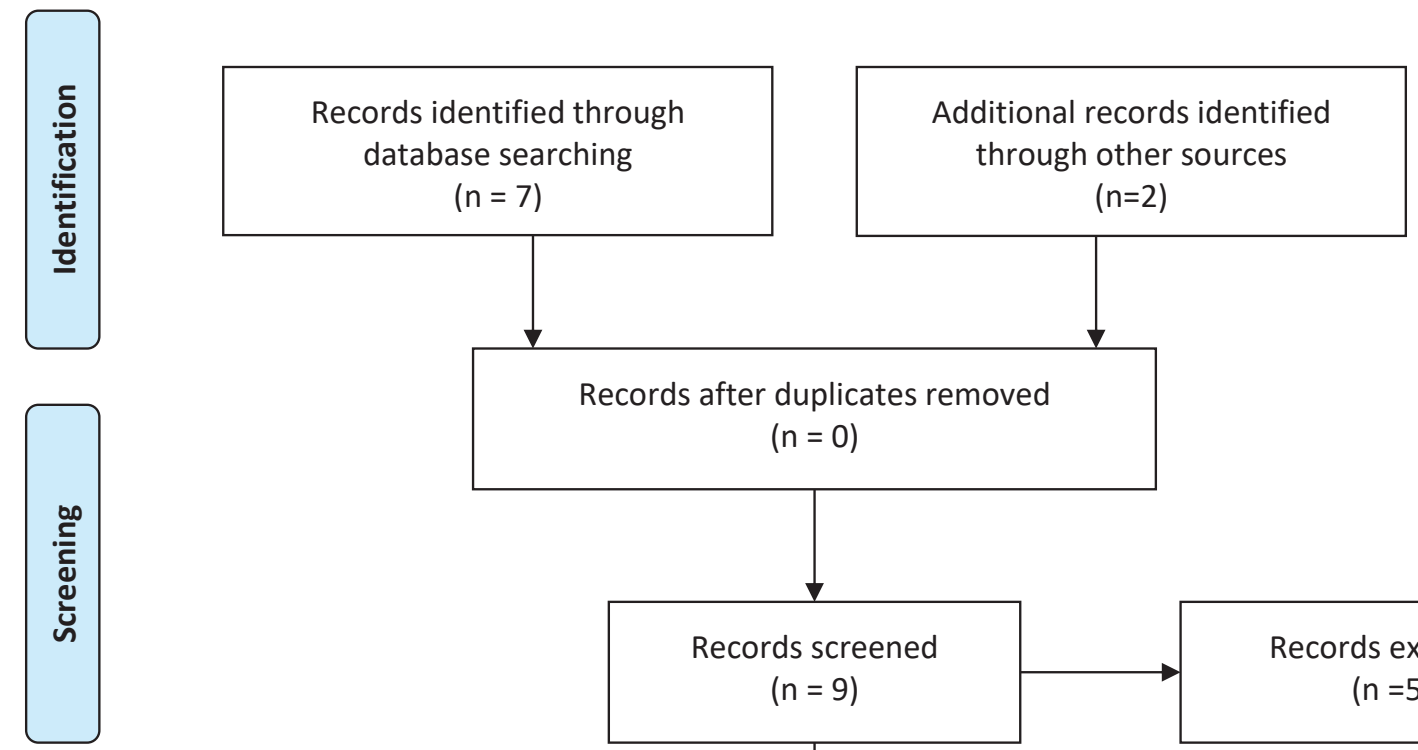

Records after duplicates removed

$(n=0)$
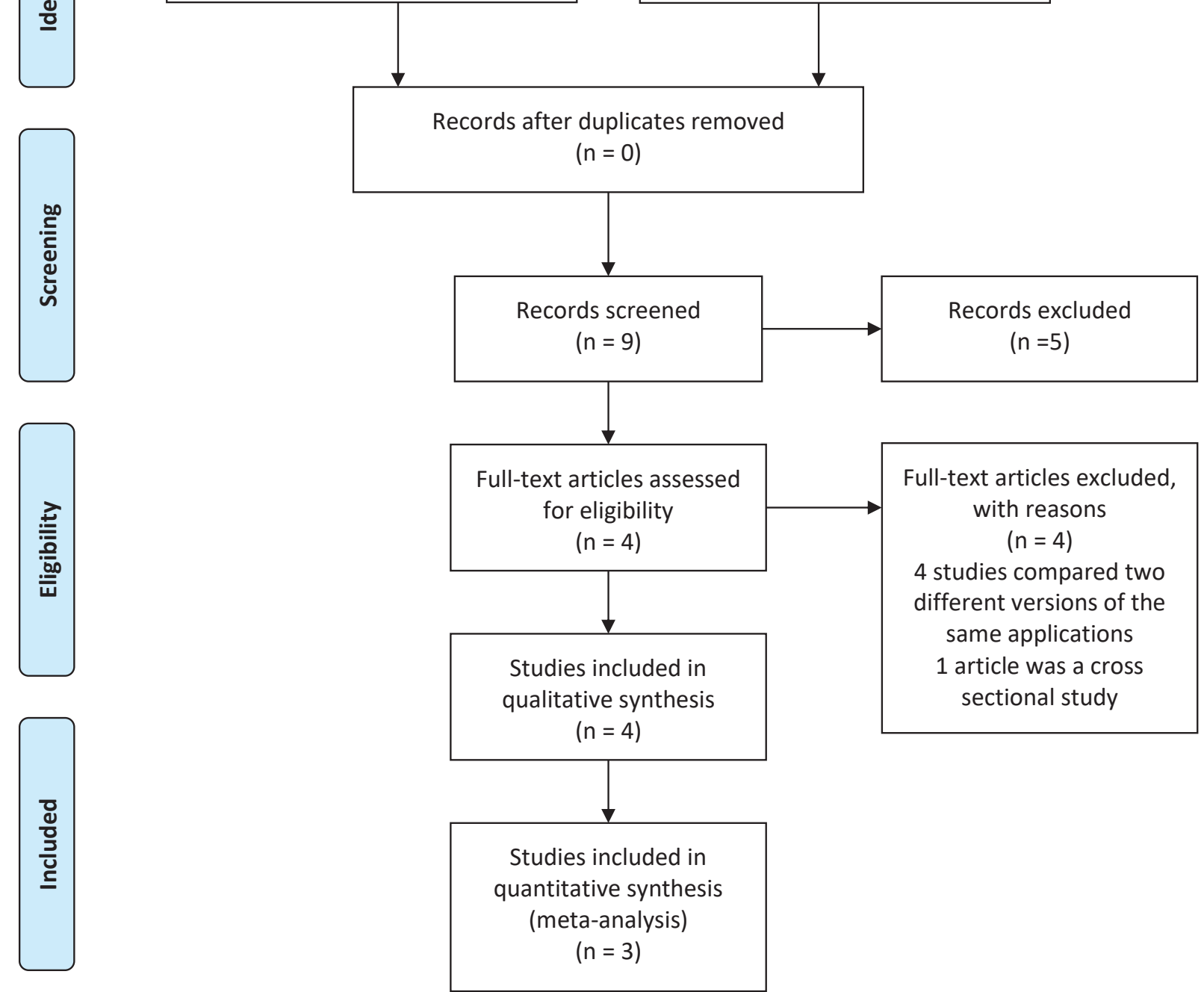

From: Moher D, Liberati A, Tetzlaff J, Altman DG, The PRISMA Group. Preferred reporting items for systematic reviews and meta-analyses: The PRISMA statement. PLoS Med. 2009;6(6):e1000097. doi:10.1371/journal.pmed.1000097. Prisma statement check list included in Supplementary file.

was conducted through a central computer-based randomization service; therefore, we could think that the randomization sequence was kept hidden until the moment of the assignment. Outcome analysis and data collection were conducted blinded to treatment allocation, but authors do not mention anything about patients. The primary outcome was selfreported continuous smoking abstinence verified by carbon monoxide breath testing at 6 months. Statistical analysis was not carried out by intention to treat. Three people from intervention group were lost in the follow-up.

In the study of Danaher et al. ${ }^{34}, 1271$ smokers (aged $\geq 18$ years) who wanted to quit were randomly assigned to: 1) MobileQuit (designed for its use in mobile devices), or 2) QuitOnline (designed for non-mobile desktop or tablet computers). Inclusion criteria were smokers who consumed $\geq 5$ cigarettes/day in the previous 6 months and those who wanted to quit smoking in next 14 days. 
Table 1. Studies reviewed

\begin{tabular}{|c|c|c|c|c|c|}
\hline Authors & Title & Sample & Intervention and comparator & $\begin{array}{l}\text { Main variables } \\
\text { and follow-up }\end{array}$ & Results \\
\hline Buller et al. ${ }^{35}$ & $\begin{array}{l}\text { Randomized Trial of } \\
\text { a Smartphone Mobile } \\
\text { Application Compared } \\
\text { to Text Messaging } \\
\text { to Support Smoking } \\
\text { Cessation }\end{array}$ & $\begin{array}{l}\text { Young adult smokers } \\
\text { aged } 18-30 \text { years } \\
(n=102) \\
\text { Of the patients, } 58- \\
66 \% \text { were planning } \\
\text { to quit smoking }\end{array}$ & $\begin{array}{l}\text { This study compares a mobile } \\
\text { application (REQ-Mobile) } \\
\text { ( } n=51) \text { with text messaging } \\
\text { to support smoking cessation } \\
\text { (on0) }(n=51)\end{array}$ & $\begin{array}{l}12 \text { weeks } \\
\text { continuous } \\
\text { abstinence } \\
\text { (Intention to treat } \\
\text { analysis) }\end{array}$ & $\begin{array}{l}\text { RED O MOBILE } \\
18 \%(7-28 \%) \\
\text { ONO } \\
31 \%(18-45 \%) \\
p=0.11\end{array}$ \\
\hline Peiris et al. ${ }^{33}$ & $\begin{array}{l}\text { A Smartphone } \\
\text { App to Assist } \\
\text { Smoking Cessation } \\
\text { Among Aboriginal } \\
\text { Australians: } \\
\text { Findings from a } \\
\text { Pilot Randomized } \\
\text { Controlled Trial }\end{array}$ & $\begin{array}{l}\text { Current Aboriginal } \\
\text { smokers (aged }>16 \\
\text { years; mean } \pm \text { SD } \\
42 \pm 14 \text { ) years), who } \\
\text { were willing to make } \\
\text { a quit attempt in the } \\
\text { next month } \\
\text { The majority of } \\
\text { patients were smokers } \\
\text { of }<20 \text { cigarettes/day }\end{array}$ & $\begin{array}{l}\text { The intervention was a } \\
\text { multifaceted Android or iOS } \\
\text { app comprising a personalized } \\
\text { profile and quit plan, text and } \\
\text { in-app motivational messages } \\
\text { (n=22). The comparator } \\
\text { was usual cessation support } \\
\text { services (24) }\end{array}$ & $\begin{array}{l}\text { Self-reported } \\
\text { continuous } \\
\text { smoking } \\
\text { abstinence, } n(\%) \\
\text { at } 4 \text { weeks and } 6 \\
\text { months visit }\end{array}$ & $\begin{array}{l}\text { Intervention } \\
\text { group } \\
0(0 \%) \\
\text { Control group } \\
0(0 \%)\end{array}$ \\
\hline Danaher et al. ${ }^{34}$ & $\begin{array}{l}\text { Outcomes and } \\
\text { Device Usage for } \\
\text { fully automated } \\
\text { internet interventions } \\
\text { designed for a } \\
\text { smartphone or } \\
\text { personal computer: } \\
\text { The Mobile Quit } \\
\text { Smoking cessation } \\
\text { randomized } \\
\text { controlled trial }\end{array}$ & $\begin{array}{l}1271 \text { smokers (aged } \\
\geq 18 \text { years; mean } \pm \\
\text { SD } 42.9 \text { (12.3) years) } \\
\text { who wanted to quit } \\
\text { in next } 14 \text { days } \\
\text { Patients were smokers } \\
\text { of } \geq 5 \text { cigarettes/ } \\
\text { day in the previous } 6 \\
\text { months }\end{array}$ & $\begin{array}{l}\text { Patients were randomly } \\
\text { assigned to MobileQuit } \\
\text { ( } n=633 \text { ) (designed for use in } \\
\text { mobile phones) or QuitOnline } \\
\text { ( } n=638 \text { ) (designed for non- } \\
\text { mobile desktop or tablet } \\
\text { computers) }\end{array}$ & $\begin{array}{l}\text { Point prevalence } \\
\text { self-reported at } 3 \\
\text { and } 6 \text { months (ITT } \\
\text { analysis) }\end{array}$ & $\begin{array}{l}3 \text { months: } \\
\text { MOBILEOUIT: } \\
131(20.7 \%) \\
\text { QUITONLINE: } \\
73(11.4 \%) \\
\text { p<0.001 } \\
6 \text { months: } \\
\text { MOBILEOUIT: } \\
\text { 156 (24.6\%) } \\
\text { QUITONLINE: } \\
123(19.3 \%) \\
\text { p=0.02 }\end{array}$ \\
\hline Baskerville et al. ${ }^{36}$ & $\begin{array}{l}\text { Effect of a Mobile } \\
\text { Phone Intervention } \\
\text { on Quitting Smoking } \\
\text { in a Young Adult } \\
\text { Population of } \\
\text { Smokers: Randomized } \\
\text { Controlled Trial }\end{array}$ & $\begin{array}{l}1599 \text { young adult } \\
\text { smokers (aged 19-29 } \\
\text { years) intending to } \\
\text { quit smoking in the } \\
\text { next } 30 \text { days }\end{array}$ & $\begin{array}{l}\text { Patients were randomized } \\
\text { to one of two groups: } \\
\text { an evidence-informed } \\
\text { smartphone app for smoking } \\
\text { cessation, Crush the Crave } \\
\text { (CTC) ( } \mathrm{n}=820) \text { and the control } \\
\text { group, an evidence-informed } \\
\text { self-help guide, On the Road } \\
\text { to Quitting (OnRO) }(\mathrm{N}=779)\end{array}$ & $\begin{array}{l}\text { Self-reported } \\
\text { continuous } \\
\text { abstinence at } 6 \\
\text { months follow-up } \\
\text { (intention to treat } \\
\text { analysis) }\end{array}$ & $\begin{array}{l}\text { CTC: } 50(6.1 \%) \\
\text { OnR0: } 60(7.7 \%) \\
p=0.28\end{array}$ \\
\hline
\end{tabular}

Participants completed an internet-based registration procedure before being assigned to a condition, via a computer-generated randomization. We could expect that the sequence of concealment was kept hidden until assignment moment. Authors do not mention anything about blinding of participants, researchers, and person responsible for statistical analysis. Statistical analysis was carried out both by intention to treat (participants who did not complete their assessments were considered to be smokers) and by complete cases. The primary variable was 7 days point prevalence at follow-up at 3 and 6 months. The protocol was published in clinicaltrials.org with number NCT01952236.

The study of Buller et al. ${ }^{35}$ was a clinical trial that included 102 adult smokers aged 18-30 years. Half were assigned to REQMobile, and the other to onQ, using an online randomization program. Patients 
Figure 2. Risk of bias

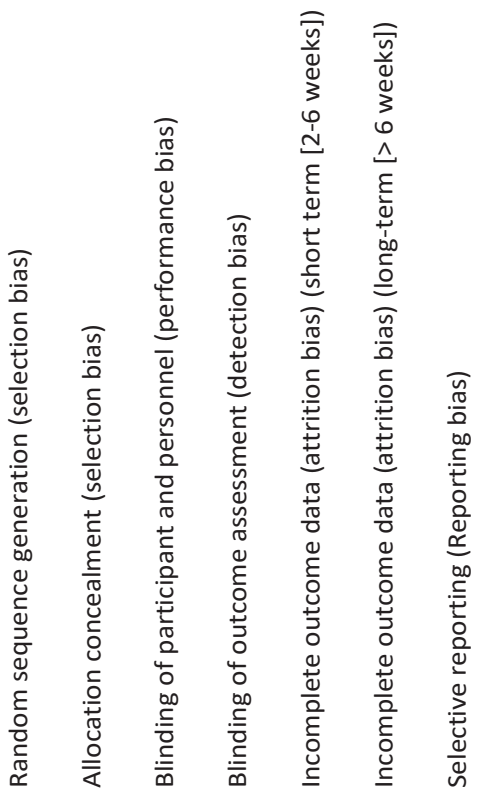

Buller et al, 2014

Peiris et al, 2019

Danaher et al, 2019

Baskerville et al, 2018

\begin{tabular}{|c|c|c|c|c|c|c|}
\hline+ & + & $?$ & $?$ & + & + & $?$ \\
\hline+ & $?$ & - & + & $?$ & $?$ & + \\
\hline+ & + & - & - & + & + & + \\
\hline+ & + & + & + & + & + & + \\
\hline
\end{tabular}

were registered into the study web site without revealing them to the investigators, but authors do not mention anything about patients. The effect of treatment was analyzed by intention to treat analysis. Patients lost to follow-up were considered as smokers for statistical analysis. In addition, the protocol of this clinical trial was not found. All smokers were invited by e-mail to complete follow-up questionnaires online at 6 and 12 weeks to collect self-reported smoking status. Quitting tobacco was measured at 6 and 12 weeks as 30 -day point-prevalence (30 PP), defined as not smoking in the past 30 days.

In the study of Baskerville et al. ${ }^{36}, 1520$ participants aged 19-29 years were eligible if they were considering quitting smoking in the next 30 days. Those referred to the study by a friend or a family member already participating in the trial, were excluded to avoid possible contamination bias. Patients that met inclusion criteria were randomly
Figure 3. Galbraith graphic

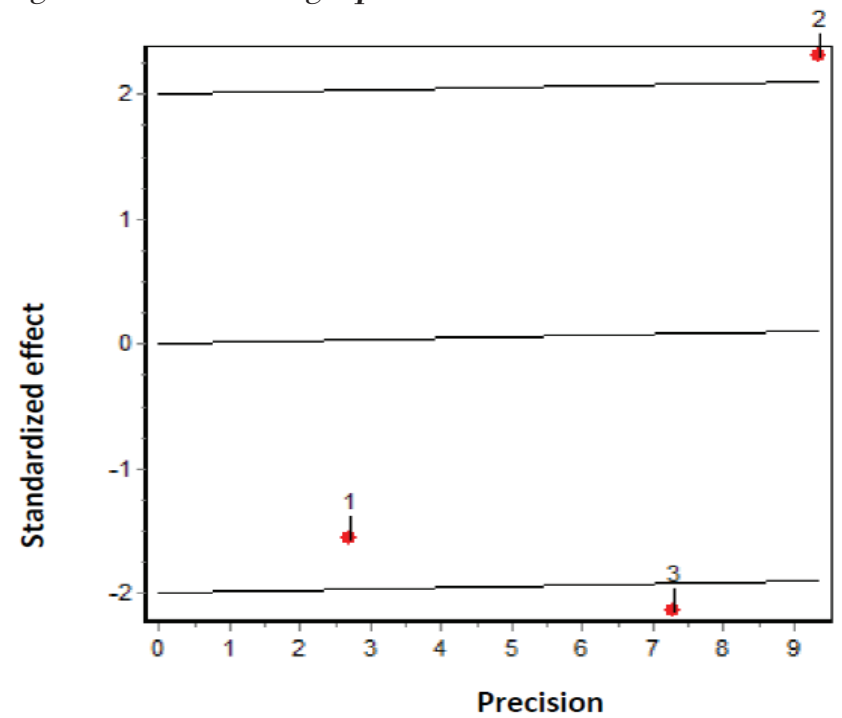

Figure 4. L'Abbé graphic

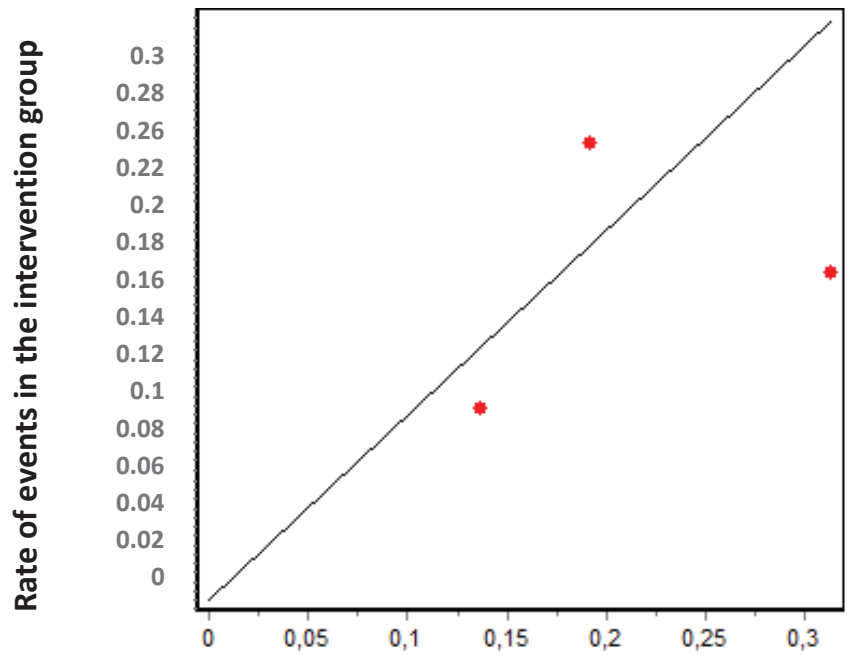

Rate of events in the control group

allocated to either the control or intervention arm, receiving a computer-generated email confirming registration. Researchers, data collectors, and participants, were blinded to the group assignments. The protocol was available before the study started, as it was registered in clinicaltrials.org with number CT01983150. The statistical analysis was carried out by intention to treat. Follow-up was conducted at 3 and 6 months, post-randomization. Quitting tobacco 
was measured with the self-reported 30-day pointprevalence abstinence (PPA) from smoking at 3 and 6 months.

\section{Heterogeneity analysis}

According to the results of the DerSimonian and Laird $^{11}$ test, there is statistical heterogeneity among the studies included in the meta-analysis $(\mathrm{P}<0.05)$. Similar results can be drawn from the graphs of Galbraith (Figure 3) and L'Abbé (Figure 4). In the former, we can observe that 2 studies are outside the confidence bands, while in the latter, we observe that points were not over a straight line. Despite the heterogeneity between studies, all were maintained in the analysis. $\mathrm{I}^{2}$ index was $76.41 \%$.

\section{Publication bias}

The p-value of the statistical tests performed was greater than 0.05 , suggesting a lack of publication bias. When analyzing the Funnel Plot and Egger graphs (Supplementary file, Figures S1 and S2), we realized that results do not follow the statistical tests. Thus, the lack of bias cannot be assumed.

\section{Estimation of pooled measurement}

The results of the studies included in the metaanalysis have been combined using a random effects model given the existence of heterogeneity. After combining the results, a total RR of 0.901 (95\% CI: 0.57-1.423) was estimated (Table 2).

\section{Sensitivity analysis}

The study with the greatest influence on the metaanalysis was that of Danaher et al. ${ }^{34}$. When this study was deleted, and the meta-analysis was repeated, the greatest variation in the estimates of the overall effect was obtained. The estimated RR decreased since a study with a beneficial effect of the intervention to be evaluated was suppressed. The sensitivity analysis showed that the measurement is robust as the direction of the effect does not change and the magnitude of the effect does it minimally (Table 3 ).

Table 2. Comparison of smartphone app versus other intervention (routine practice, text messaging, app for computer or tablet)

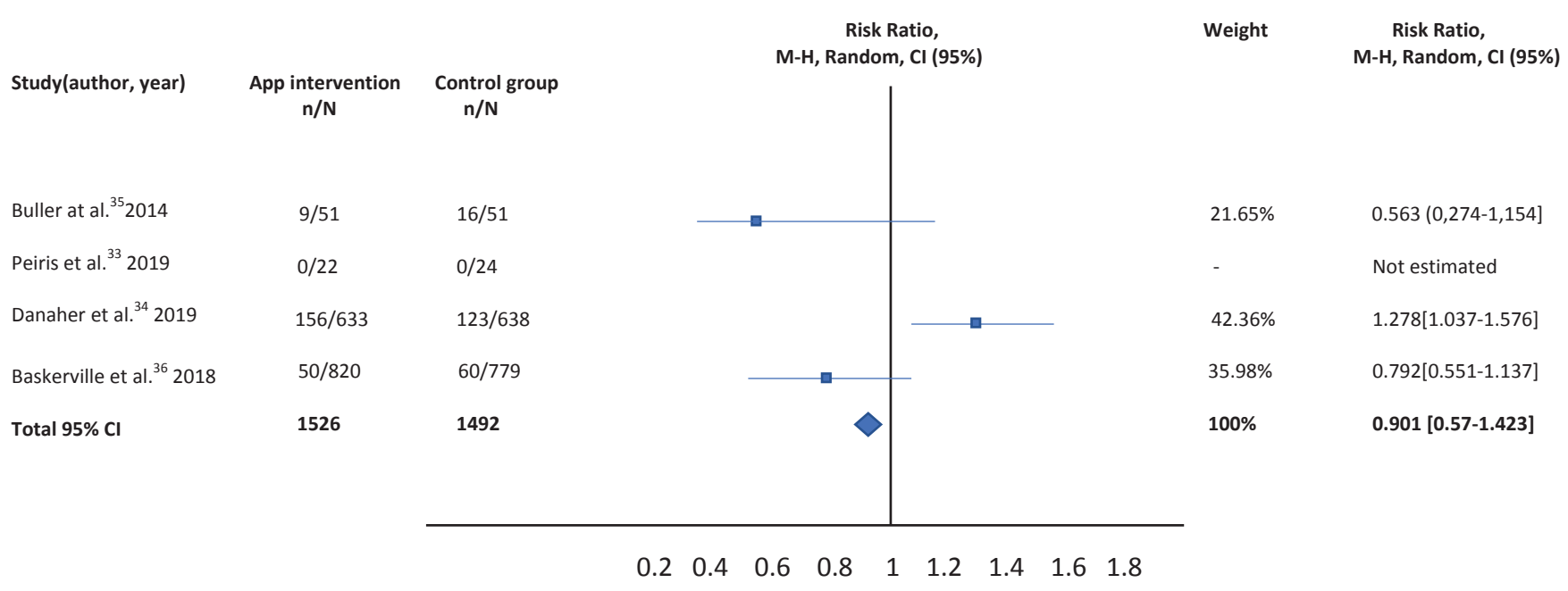


Table 3. Comparison of smartphone app versus other intervention: Sensitivity analysis

\begin{tabular}{|c|c|c|c|c|}
\hline & & $\begin{array}{c}\text { Risk Ratio, } \\
\text { M-H, Random, Cl (95\%) }\end{array}$ & Weight & $\begin{array}{l}\text { Risk Ratio, } \\
\text { M-H, Random, Cl (95\%) }\end{array}$ \\
\hline Study (author, year) & $\begin{array}{l}\text { App intervention } \\
\mathrm{n} / \mathrm{N}\end{array}$ & & & \\
\hline Buller at al. ${ }^{35} 2014$ & 2870 & 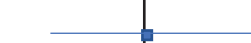 & 14.38 & $1.030(0.646-1.644)$ \\
\hline Danaher et al. ${ }^{34} 2019$ & 1701 & $\square$ & -17.98 & $0.739(0.535-1.021)$ \\
\hline Baskerville et al. ${ }^{36} 2018$ & 1373 & $\square$ & 1.48 & $0.914(0.414-2.016)$ \\
\hline Total 95\% Cl & & $>$ & & $0.901(0.57-1.423)$ \\
\hline
\end{tabular}

\section{DISCUSSION}

After combining the results of the studies included in the meta-analysis, no statistically significant differences are observed between the effectiveness of the app as a tool to quit smoking and the rest of the therapeutic options evaluated. Relative risk for smoking cessation using a mobile application for smartphones versus other type of interventions was 0.901 (95\% CI: 0.57-1.423). Studies have been combined using a random effects model given the absence of homogeneity among the included studies.

\section{Strengths and limitations}

There are several limitations to the study. First, the heterogeneity is not only statistical but also clinical, since the populations of the included studies are different from each other. Furthermore, the interventions and follow-up periods were not the same for all studies. Indeed, Buller et al. ${ }^{35}$ did a maximum follow-up of 12 weeks, while in the remaining studies it was 6 months. In relation to the main variable, Danaher et al. ${ }^{34}$ measured the 7 days point prevalence whereas the remaining studies ${ }^{35,36}$ evaluated the 30 days point-prevalence abstinence. However, this does not affect our results, because those who have smoked in the past 30 days have also smoked in the past 7 days.

Despite the limitations, the present meta-analysis study has several important strengths. First, an analysis of the risk of bias of the four studies was carried out, showing that the risk of bias in most domains is not high. Second, an analysis of publication bias was performed, detecting that there is, indeed, a risk of publication bias, due to the recent boom of smartphone applications. Accordingly, many ongoing studies will provide results and conclusions within 2-3 years ${ }^{37-40}$ and a new meta-analysis study will be welcome. Third, the sensitivity analysis showed the robustness of the measurement with the available data, with no significant changes in the magnitude of the combined measurement when repeating the meta-analysis by deleting a study each time. Fourth, another meta-analysis evaluating the effectiveness of the use of a mobile application compared to other alternatives has not been found in the literature. The only exception is the study of Wittaker et al. ${ }^{41}$. However, these authors performed different meta-analysis depending on the type of intervention while our objective was to compare the effectiveness of the use of a mobile application against any existing intervention to quit smoking.

\section{Future directions}

The internet has revolutionized the computer and communications world. Indeed, new forms of technology have been generated in almost all aspects of life ${ }^{42}$. New mobile devices have appeared on the market. As a consequence, the number of downloads increased in parallel with the number of mobile phone users. For example, in Spain, it is estimated that 3.8 million applications are downloaded every day. In fact, each smartphone has an average of 39 applications ${ }^{43}$. 
We are facing a global new revolution in medicine. Almost $90 \%$ of the world's population could benefit from the opportunities that mobile technologies represent at a relatively low $\operatorname{cost}^{44}$. A total of $36 \%$ of all smartphone owners have mHealth apps on their devices. Interestingly, among those using apps, $60 \%$ use mHealth apps in achieving health behavior goals $^{45}$.

\section{CONCLUSIONS}

According to the results presented, we are not able to claim that apps are effective for quitting tobacco. As the boom in mobile applications has been very recent, there are very few clinical trials that evaluate their effectiveness against other types of smoking cessation interventions. Even though some studies with protocols of clinical trials have been published to evaluate the effectiveness of mobile applications compared to other alternatives ${ }^{36-39}$, the trials are still in progress, and therefore the results have not been included in the meta-analysis. In addition, as the adherence to smoking cessation treatment guidelines is generally low, this may potentially have a negative impact on the effectiveness of apps ${ }^{46}$. On the other hand, and focusing on the usefulness of health app use, there are several patient characteristics that make the difference. Age and education level are significantly related to mobile health app use. The young and more highly educated are more likely to use them. Although gender is not associated with general mobile health app use, men are more likely to use fitness apps than women, and women are more likely to use nutrition, self-care, and reproductive health apps ${ }^{47}$. Last, but not least, as it is well known that changes promoted by health advice do not last over time (no more than six months), it would be convenient to design clinical trials with follow-up periods longer than 6 months, at least one year, to have a more suitable evaluation of the app ${ }^{47}$. Is investing in promoting the use of mobile applications justified, or do we have to continue using tools that have showed effectiveness until now ${ }^{7}$. In summary, it is necessary to go deeper into the characteristics of each app and into the features of potential users in order to try to maximize adherence and success.

\section{REFERENCES}

1. Anthonisen NR, Skeans MA, Wise RA, et al. The effects of a smoking cessation intervention on 14.5-year mortality: a randomized clinical trial. Ann Intern Med. 2005;142(4):233239. doi:10.7326/0003-4819-142-4-200502150-00005

2. Jha P, Ramasundarahettige C, Landsman V, et al. 21stcentury hazards of smoking and benefits of cessation in the United States. N Engl J Med. 2013;368(4):341-350. doi:10.1056/NEJMsa1211128

3. World Health Organization. Tobacco. https://www.who. int/news-room/fact-sheets/detail/tobacco. Updated May 27, 2020. Accessed August 9, 2019.

4. Tverdal A, Bjartveit K. Health consequences of reduced daily cigarette consumption. Tob Control. 2006;15(6):472-480. doi:10.1136/tc.2006.016246

5. He Y, Jiang B, Li LS, et al. Changes in Smoking Behavior and Subsequent Mortality Risk During a 35-Year Follow-up of a Cohort in Xi'an, China. Am J Epidemiol. 2014;179(9):1060-1070. doi:10.1093/aje/kwu011

6. Chaiton M, Diemert L, Cohen JE, et al. Estimating the number of quit attempts it takes to quit smoking successfully in a longitudinal cohort of smokers. BMJ Open. 2016;6(6):e011045. doi:10.1136/bmjopen-2016-011045

7. Cobos-Campos R, Apiñaniz Fernández de Larrinoa A, Arantza Sáez de Lafuente Morínigo A, Parraza Diez N, Aizpuru Barandiaran F. Effectiveness of text messaging as an adjuvant to health advice in smoking cessation programs in primary care. A randomized clinical trial. Nicotine Tob Res. 2017;19(8):901-907. doi:10.1093/ntr/ntw300

8. Rondina R de C, Gorayeb R, Botelho C. Psychological characteristics associated with tobacco smoking behavior. J Bras Pneumol. 2007;33(5):592-601. doi:10.1590/s1806-37132007000500016

9. Siu AL. Behavioral and Pharmacotherapy Interventions for Tobacco Smoking Cessation in Adults, Including Pregnant Women: U.S. Preventive Services Task Force Recommendation Statement. Ann Intern Med. 2015;163(8):622. doi:10.7326/M15-2023

10. Park ER. Behavioral approaches to smoking cessation. Uptodate. https://www.uptodate.com/contents/ behavioral-approaches-to-smoking-cessation. Accessed June 30, 2018.

11. Whittaker R, McRobbie H, Bullen C, Rodgers A, Gu Y. Mobile phone-based interventions for smoking cessation. Cochrane Database Syst Rev. 2016;4:CD006611. doi:10.1002/14651858.CD006611.pub4

12. Chen YF, Madan J, Welton N, et al. Effectiveness and cost-effectiveness of computer and other electronic aids for smoking cessation: a systematic review and network meta-analysis. Health Technol Assess. 2012;16(38):1205. doi:10.3310/hta16380

13. Taylor GMJ, Dalili MN, Semwal M, Civljak M, Sheikh A, Car J. Internet-based interventions for smoking cessation. Cochrane Database Syst Rev. 2013;7:CD007078. doi:10.1002/14651858.CD007078.pub5

14. Matkin W, Ordóñez-Mena JM, Hartmann-Boyce J. Telephone counselling for smoking cessation. 
Cochrane Database Syst Rev. 2019;5(5):CD002850. doi:10.1002/14651858.CD002850.pub4

15. Choi J, Noh GY, Park DJ. Smoking Cessation Apps for Smartphones: Content Analysis With the Self-Determination Theory. J Med Internet Res. 2014;16(2):e44. doi:10.2196/jmir.3061

16. Schwartz RP, Gryczynski J, Mitchell SG, et al. Computerized versus in-person brief intervention for drug misuse: A randomized clinical trial. Addiction. 2014;109(7):1091-1098. doi:10.1111/add.12502

17. Abroms LC, Padmanabhan N, Thaweethai L, Phillips T. iPhone apps for smoking cessation: a content analysis. Am J Prev Med. 2011;40(3);279-285. doi:10.1016/j.amepre.2010.10.032

18. Abroms LC, Lee Westmaas J, Bontemps-Jones J, Ramani R, Mellerson J. A content analysis of popular Smartphone apps for smoking cessation. Am J Prev Med. 2013;45(6):732-736. doi:10.1016/j.amepre.2013.07.008

19. Haskins BL, Lesperance D, Gibbons P, Boudreaux ED. A systematic review of smartphone applications for smoking cessation. Transl Behav Med. 2017;7(2):292299. doi:10.1007/s13142-017-0492-2

20. ITU releases 2015 ICT figures: Statistics confirm ICT revolution of the past 15 years. https://www.itu.int/net/ pressoffice/press_releases/2015/17.aspx. Published May 26, 2015. Accessed October 10, 2019.

21. Wallace S, Clark M, White J. 'It's on my iPhone': attitudes to the use of mobile computing devices in medical education, a mixed-methods study. BMJ Open. 2012;2(4):e001099. doi:10.1136/bmjopen-2012-001099

22. Aungst TD. Medical applications for pharmacists using mobile devices. Ann Pharmacother. 2013;47(7-8):10881095. doi:10.1345/aph.1S035

23. Krebs P, Duncan DT. Health App Use Among US Mobile Phone Owners: A National Survey. JMIR Mhealth Uhealth. 2015;3(4):e101. doi:10.2196/mhealth.4924

24. Bol N, Helberger N, Weert JCM. Differences in mobile health app use: A source of new digital inequalities?. The Information Society. 2018;34(3):183-193. doi:10.1080/01972243.2018.1438550

25. Chevalking SKL, Ben Allouch S, Brusse-Keizer M, Postel MG, Pieterse ME. Identification of Users for a Smoking Cessation Mobile App: Quantitative Study. J Med Internet Res. 2018;20(4):e118. doi:10.2196/jmir.7606

26. Mikulic M. Number of downloads of pharmaceutical and mHealth apps worldwide in 2016. Statista. https://www. statista.com/statistics/808673/worldwide-downloads-ofpharma-and-mhealth-apps/. Published March 29, 2018. Accessed August 12, 2019.

27. Cohrane Training. Cochrane Handbook for Systematic Reviews of Interventions. Version 5.1.0. https://training. cochrane.org/cochrane-handbook-systematic-reviewsinterventions. Updated March, 2011. Accessed November 9, 2019.

28. DerSimonian R, Laird N. Meta-analysis in clinical trials. Control Clin Trials. 1986;7(3):177-188. doi:10.1016/0197-2456(86)90046-2

29. Fleiss JL. The statistical basis of meta-analysis. Stat Methods Med Res. 1993;2(2):121-149. doi:10.1177/096228029300200202

30. Galbraith R. A note on graphical presentation of estimated odds ratios from several clinical trials. Stat Med. 1988;7(8):889-894. doi:10.1002/sim.4780070807

31. L'Abbe KA, Detsky AS, O'Rourke K. Meta-analysis in clinical research. Ann Intern Med. 1987;107(2):224-233. doi:10.7326/0003-4819-107-2-224

32. Dinnes J, Deeks J, Kirby J, Roderick P. A methodological review of how heterogeneity has been examined in systematic reviews of diagnostic test accuracy. Health Technol Assess. 2005;9(12):1-113. doi:10.3310/hta9120

33. Peiris D, Wright L, News M, et al. Smartphone app to assist smoking cessation among aboriginal Australians: findings from a pilot randomized controlled trial. JMIR Mhealth Uhealth. 2019;7(4):e12745. doi:10.2196/12745

34. Danaher BG, Tyler MS, Crowley RC, Brendryen H, Seeley JR. Outcomes and device usage for fully automated internet interventions designed for a smartphone or personal computer: the mobileQuit smoking cessation randomized controlled trial. J Med Internet Res. 2019;21(6):e13290. doi:10.2196/13290

35. Buller DB, Borland R, Bettinghaus EP, Shane JH, Zimmerman DE. Randomized trial of a smartphone mobile application compared to text messaging to support smoking cessation. Telemed J E Health. 2014;20(3):206214. doi:10.1089/tmj.2013.0169

36. Baskerville NB, Struik LL, Guindon GE, et al. Effect of a mobile phone intervention of quitting smoking in a young adult population of smokers: randomized controlled trial. JMIR Mhealth Uhealth. 2018;6(10):e10893. doi:10.2196/10893

37. Cambon L, Bergman P, Le Faou A, et al. Study protocol for a pragmatic randomised controlled trial evaluating efficacy of a smoking cessation e-'Tabac Info Service': ee-TIS trial. BMJ Open. 2017;7(2):e013604. doi:10.1136/bmjopen-2016-013604

38. BinDhim NF, McGeechan K, Trevena L. Assessing the effect of an interactive decision-aid smartphone smoking cessation application (app) on quit rates: a double-blind automated randomised control trial protocol. BMJ Open. 2014;4(7):e005371. doi:10.1136/bmjopen-2014-005371

39. Lüscher J, Berli C, Schwaninger P, et al. Smoking cessation with smartphone applications (SWAPP): study protocol for a randomized controlled trial. BMC Public Health. 2019;19(1):1400. doi:10.1186/s12889-019-7723-z

40. Chu KH, Escobar-Viera CG, Matheny SJ, Davis EM, Primack BA. Tobacco cessation mobile app intervention (Just Kwit! study): protocol for a pilot randomized controlled pragmatic trial. Trials. 2019;20(1):147. doi:10.1186/s13063-019-3246-2

41. Whitakker R, McRobbie H, Bullen C, Rodgers A, Gu Y, Dobson R. Mobile phone text messaging 
and app-based intervention for smoking cessation. Cochrane Database Syst Rev. 2019;10:CD006611. doi:10.1002/14651858.CD006611.pub5

42. Van De Belt TH, Engelen LJ, Berben SA, Schoonhoven L. Definition of Health 2.0 and Medicine 2.0: a systematic review. JMIR. 2010;12(2):e18. doi:10.2196/jmir.1350

43. Fundación Telefónica. La Sociedad de la Información en España 2013. https://www.fundaciontelefonica. $\mathrm{com} /$ cultura-digital/publicaciones/sociedad-de-lainformacion/. Published 2014. Accessed February 11, 2020.

44. World Health Organization. Mhealth New horizons for health through mobile technologies. In: Global Observatory for ehealth series, volume 3. https://www. who.int/goe/publications/goe_mhealth_web.pdf?. Accessed February 11, 2020.

45. Bhuyan SS, Lu N, Chandak A, et al. Use of Mobile Health Applications for Health-Seeking Behavior Among US Adults. J Med Syst. 2016;40(6):153. doi:10.1007/s10916-016-0492-7

46. Rajani NB, Weth D, Mastellos N, and Filippidis FT. Adherence of popular smoking cessation mobile applications to evidence-based guidelines. BMC Public Health. 2019;19:743. doi:10.1186/s12889-019-7084-7

47. Rise MB, Pellerud A, Rygg LØ, Steinsbekk A. Making and Maintaining Lifestyle Changes after participating in Group Based Type 2 Diabetes Self-Management Educations: A Qualitative Study. Plos One. 2013;8(5):e64009. doi:10.1371/journal.pone.0064009

CONFLICTS OF INTEREST

The authors have completed and submitted the ICMJE Form for Disclosure of Potential Conflicts of Interest and none was reported.

\section{FUNDING}

There was no source of funding for this research.

\section{AUTHORS' CONTRIBUTIONS}

$\mathrm{RC}$ performed the meta-analysis and written the manuscript. GO has thoroughly reviewed and approved the final version of the manuscript. ASL, $N P, A A$ and IP have read and approved the final version.

PROVENANCE AND PEER REVIEW

Not commissioned; externally peer reviewed. 